EDITORIAL

\title{
2nd Special Issue: Tackling key immunological and immuno-dermatological pathways and their link to treatment options
}

\author{
DANIEL BODA ${ }^{1,2}$ \\ ${ }^{1}$ Research Laboratory, 'Carol Davila' University of Medicine and Pharmacy, 050474 Bucharest; \\ ${ }^{2}$ Department of Dermatology, 'Prof. N. Paulescu' National Institute of Diabetes, \\ Nutrition and Metabolic Diseases, 79811 Bucharest, Romania
}

Received June 30, 2020; Accepted July 7, 2020

DOI: $10.3892 /$ etm.2020.8977

Immunological pathways have been extensively studied over the past 30 years as key processes of the pathogenesis and outcomes of several immunological and dermatological diseases and have been linked to new therapeutic treatment options. Thus, the current special issue focuses on reviews, original articles and case presentations in immunology and immuno-dermatology, in order to partially elucidate those pathways and processes.

The main physiological and/or pathological conditions covered in the current special issue were immunological phenomena involved in: COVID-19 infection, CMV infection, immunological aspects in pregnancy, psychiatric disorders, ophthalmology, diabetes mellitus and its associated diseases, autoimmune thyroiditis.

The COVID pandemic has widely monopolized public attention, as well as health providers in the first part of 2020.

The review by Tanasa et al (1) entitled 'Anosmia and ageusia associated with coronavirus infection (COVID-19) what is known?' summarizes the existing data regarding the association of anosmia and ageusia with the SARS-CoV-2 infection. It also aims to describe these manifestations, particularly in the clinical setting of all symptomatic patients.

Urticarial hives, mast cell activation and respiratory allergies are frequent aspects encountered during the summer months. In their study entitled 'Mast cell activation syndromes - evaluation of current diagnostic criteria and laboratory tools used in clinical practice (Review)', Leru et al (2) aimed to

Correspondence to: Dr Daniel Boda, Research Laboratory, 'Carol Davila' University of Medicine and Pharmacy, 8 Eroii Sanitari Avenue, 050474 Bucharest, Romania

E-mail: daniel.boda@yahoo.com

Key words: immunology, immuno-dermatology, infection, pregnancy, psychiatric disorders, ophthalmology, diabetes mellitus review the actual diagnosis criteria of mast cell activation syndromes, some relevant clinical aspects and laboratory tools used in clinical practice.

In their study entitled 'Relationship between chemokines and $\mathrm{T}$ lymphocytes in the context of respiratory allergies (Review)', Berghi et al (3). presented the interactions between chemokines and Th lymphocytes in the context of Th2/ non-Th2 endotype classification of respiratory allergies.

In their review entitled 'Immunologic approaches and therapy in burns (Review)', Boldeanu et al (4) aimed to identify novel methods with which to modulate the defense capacity, to prevent the complications of abnormal immune response and to treat efficiently the burn injuries. They also discussed the link between inflammasomes, particularly the 'Nucleotidebinding domain and leucine-rich repeat protein 3 (NLRP3) inflammasome' and the defense capacity of the innate immune system.

In their study entitled 'Postanesthetic skin erythema due to succinylcholine versus atracurium' Ciobotaru et al (5) aim to compare the side-effects of systemic anesthesia with succinylcholine vs. atracurium and in the role of Mas-Related G-Protein-coupled Receptor X2 receptor (MRGPRX2), considered as a target for some neuromuscular blockers such as atracurium, rocuronium or fluoroquinolone, resulting in pseudoallergic or anaphylactoid reactions.

In addition, as tick bites are very frequent during the summer season, the pathologies associated with these are always a matter of concern. In their study entitled 'Etiologic role of Borrelia burgdorferi in morphea: A case report', Sandru et al (6) aimed to discuss the main mechanisms involved in the etiophatogenesis of morphea, as well as the therapeutic options. They present a case of a 60 -year-old woman, who was referred to their clinic for an erythematousviolaceus, asymptomatic eruption, located on the trunk and legs, for approximately 2 months. The patient's medical history revealed an infection with Borrelia 1 year prior. After the diagnosis of morphea was established, and with systemic therapy (corticosteroids and methotrexate), the evolution was favorable. 
Vrinceanu et al (7) present a case of a male with drug rash with eosinophilia and systemic symptoms (DRESS) syndrome induced by carbamazepine intake.

Surlin et al (8) aimed to assess whether the increased production of interleukin (IL)-1 $\alpha$ and IL-1 $\beta$, as a result of chronic hepatic inflammation, was the expression of the negative impact on the severity and evolution of periodontal disease. Positive associations were found between gingival fluid IL-1 $\alpha$ and $-1 \beta$ levels and certain clinical periodontal parameters or the age of the viral hepatitis $\mathrm{C}$ diagnosis, in periodontitis patients with chronic hepatitis $\mathrm{C}$. The chronic hepatic inflammation may have an important additional negative impact on the periodontal status, as both inflammatory reactions seem to be promoted by common pro-inflammatory cytokines.

Bica et al (9) wrote a review regarding 'Non-alcoholic fatty liver disease: A major challenge in type 2 diabetes mellitus (Review)' highlighting that patients with non-alcoholic steatohepatitis (NASH) and T2DM have an increased mortality and morbidity. Therefore, they generate substantial healthcare costs. Thus, the objectives in NAFLD and NASH therapy are to reduce disease activity, to attenuate the progression of fibrosis and to reduce the risk factors.

In 'Stomach 'tastes' the food and adjusts its emptying: A neurophysiological hypothesis (Review)', Papacocea et al (10) demonstrated that the presence of taste receptors and their secondary messengers in the stomach raised the possibility that the stomach may play a role in food 'tasting' and consequently, it may initiate specific adaptations of its secretory and motor function. In addition, a variety of chemical mediators are able to modulate the activity of the enteric nervous system (ENS) and to influence both secretory and motor functions of the stomach.

In 'Metabolic and cardiovascular benefits of GLP-1 agonists, besides the hypoglycemic effect (Review)', Iorga et al (11) present the cardiovascular and metabolic benefits of GLP-1 RAs versus the placebo in patients with type 2 diabetes. The international guidelines recommend using GLP-1 RAs as first-line therapy in type 2 diabetes patients with high cardiovascular risk or as first-line agents in patients intolerant to metformin.

In 'New oral anticoagulants - possible extension to other indications (Review)', Laslo et al (12) detail the clinical indications for new oral anticoagulants: Prevention and treatment of venous thromboembolism, the prevention of stroke in patients with nonvalvular atrial fibrillation, postoperative venous thromboprophylaxis after hip replacement surgery, atherothrombotic events in adults with coronary artery disease and/ or peripheral artery disease, reasonable choice of anticoagulation for patients with cancer.

'Benefits of erectile function recovery programs after radical prostatectomy' is a review by Marcu et al (13) underlining the impact of phosphodiesterase 5 inhibitors in terms of preventing the fibrotic changes that are responsible for the irreversible erectile dysfunction after prostatectomy. The general opinion is that an erectile function recovery process should be started as soon as possible after surgery to prevent the negative effects of neuropraxia.

Several conditions are of critical value in influencing the outcome of pregnancies as well as the wellbeing of the fetus. Thus, Voicu et al (14) postulated in 'Maternal inherited thrombophilia and pregnancy outcomes' that maternal thrombophilia may be a risk factor for preeclampsia and intrauterine growth retardation. Therefore, they studied 459 pregnant women and demonstrated that the type of thrombophilic mutation most commonly found was the MTHFR mutation (25.7\%), followed by the prothrombin gene mutation (20.9\%) and the Leiden factor V mutation (15.7\%). In addition, $15.03 \%$ patients had been diagnosed with preeclampsia and $6.75 \%$ of the pregnant women had IUGR fetuses.

In the study entitled 'Unfavorable influence of the prematurity on the neonatal prognostic of small for gestational age fetuses', Turcan et al (15) aimed to evaluate the neonatal prognosis of preterm births with and without growth restriction and term births with growth restriction in order to improve decisional accuracy regarding the termination of pregnancy.

Bohiltea et al (16) wrote an original study 'Impact of obesity on the prognosis of hypertensive disorders in pregnancy', analyzing cases of pregnancy and postpartum complicated by pathologies related to placental dysfunction: Infections during pregnancy, diabetes, thrombophilia, pregnancy obtained by in vitro fertilization, abnormal adherence of the placenta, obesity, multiple pregnancy, the presence of an earlier hepatic, endocrine, renal, cardiac or autoimmune pathology, and the existence of an uterine malformation. Thus, obese patients have a higher risk of having moderate preeclampsia, following gestational hypertension and finally severe preeclampsia.

In their original study entitled 'Prognosis of autoimmune thyroid disease associated with hereditary thrombophilia during pregnancy', Păuleț et al (17) assessed the connection between acute and chronic thyroiditis and inherited thrombophilia and their potential implications in pregnancy. Besides the well-accounted impact of hereditary thrombophilia during pregnancy, which is emphasized in their research by the incidence of moderate preeclampsia, they also account for a direct and statistically significant relationship between thrombophilia and moderate intensity autoimmune diseases (including autoimmune thyroiditis). In addition, if these two pathologies are combined, there is an increased risk of the presence of another autoimmune disease.

In their study entitled, 'Conservative in utero treatment of fetal dyshormonogenetic goiter with levothyroxine, a systematic literature review', Nemescu et al (18) assessed the feasibility, safety, and effectiveness of the conservative in utero treatment of fetal goitrous hypothyroidism and confirm the feasibility of levothyroxine.

In their study entitled 'Cytomegalovirus seroprevalence in pregnant women in the western region of Romania: A largescale study', Gorun et al (19) analyzed the CMV seroprevalence according to demographic features of pregnant women in western Romania, as well as the evolution of CMV immunity in two time intervals. The seroprevalence was higher in women from rural areas compared to those from urban areas. These results show that the western region of Romania has a low-risk profile for primary CMV infection during pregnancy due to a large number of seropositive women. However, this risk has increased over the past 10 years, from 5.4 to $8.2 \%$, which may indicate the need to implement a national screening program.

In their original study entitled 'A retrospective study regarding the method of delivery of adolescents in a Romanian 
Hospital', Uzunov et al (20) aimed to analyze the pregnancyrelated conditions, the way of delivery and the neonatal outcome of all the patients who delivered in the Department of Obstetrics and Gynecology of University Emergency Hospital in Bucharest, a tertiary unit in Romania over a period of 5 years, on a group of 686 patients. As regards their results, 464 of the 686 patients did not undergo any medical visits during pregnancy. A total of $52.76 \%$ of the patients delivered by caesarean section. The most frequent indications for caesarean extraction, in both analyzed groups, were cephalopelvic disproportion, fetal distress and uterine scar following caesarean section. The lack of specific protocols regarding the ante-, peri- and post-natal management of adolescents is probably the cause of the alarming increasingly number of patients pertaining to this group who deliver by caesarean section.

Navolan et al (21) performed an original study, entitled 'Comparison of early pregnancy serum concentration of neopterin, neopterin/creatinine ratio, $\mathrm{C}$-reactive protein, and chitotriosidase, in pregnant women with birth at term and spontaneous preterm birth', comparing the course of inflammatory markers (IM) [neopterin (Neo), neopterin/creatinine ratio $(\mathrm{Neo} / \mathrm{Cre}), \mathrm{C}$ reactive protein $(\mathrm{CRP})$, and chitotriosidase (Chito)] serum concentration in the early pregnancy of pregnant women with birth at term (BT) and preterm birth (PB).

In their study entitled 'Variation of total serum sialic acid concentration in postmenopausal women', Olaru et al (22) assessed the age-related changes in serum sialic acid levels in 97 elderly subjects, including men and postmenopausal women. A significant increase in serum levels of sialic acid in postmenopausal women and a significant decrease in men were observed in an age-dependent manner. Furthermore, in postmenopausal women, positive correlations were observed between the total serum sialic acid level and age in all decades subgroups. The total serum concentration of sialic acid in the postmenopausal women group exhibited a particular pattern; significantly lower levels were observed in subjects in the intermediate postmenopausal period compared with those in the first decade of post menopause and then higher levels again as the women get older (late climax), thus the concentration increased according to the age of the individual.

In their original study entitled 'Dietary behavior during pregnancy', Iordachescu et al (23) addressed a questionnaire to 100 pregnant women in their third trimester of pregnancy in order to evaluate a possible correlation between the age and the dietary behavior and lifestyle of a pregnant woman. Furthermore, the maternal compliance with dietary recommendations in pregnancy and the exposure to various risks due to unhealthy nutrition were also analyzed in the study.

In the case presentation 'Anatomical and functional changes of uterine arteries circulation after modified laparoscopic transabdominal cerclage in pregnancy: A case report and review of the literature', Nemescu et al (24) described a safer approach for the treatment of cervical insufficiency in pregnancy, with the cerclage tape placed lateral to the uterine vessels. They describe and review the evolution of a woman who successfully underwent an LTAC at 12 weeks of gestation, whose fetus developed growth retardation after 32 weeks.

Zvanca et al (25), in their study entitled 'Posterior fossa assessment in the axial view of the head at 11-14 weeks of gestation in normal and aneuploid fetuses', aimed to identify an easy, reproducible method to exam the posterior fossa. They found a positive correlation between the first trimester and second-trimester CM diameter and between the first-trimester fourth ventricle and second trimester TCD. There is an inverse correlation between the first trimester $\mathrm{CM}$ and second trimester TCD. The measurements of different components of the posterior fossa in the first trimester cannot predict the size of CM and TCD in the second trimester. The presence of the three hypoechoic structures (cerebral peduncles, fourth ventricle, and CM) separated by two hyperechoic lines is easy to observe and measure.

Nemescu et al (26) performed a prospective study entitled 'Reference ranges for the fetal mesencephalon to occiput measurement at 11 to $13+6$ weeks of gestation' in order to obtain a quantitative description of the normal position of the fetal midbrain in the first trimester, by defining the reference ranges for the mesencephalon to the occipital bone distance, in the axial plane. This was a prospective study that included normal fetuses screened between 11 and 13 weeks of gestation. They described a simple measurement between the midbrain and the occipital bone, obtained in the same axial view. It increases linearly with advancing gestation. The integration of this measurement into the routine ultrasound screening in association with the 'crash sign' and recognizing the lower extreme values could lead to an early diagnosis of open spina bifida.

In their study entitled 'Heart valve mechanical prosthesis: The perfect match until it is not - A case report', Movileanu et al (27) present a case report that discusses the disadvantages and possible fatal complications of the association between mechanical valves and severe thrombocytopenia.

In 'Use of prokinetics and other quality factors in capsule endoscopy - old and new insights (Review)', Costache et al (28) debate about prokinetics - diverse pharmacological substances to increase the success rate of capsule endoscopy.

Iliesiu et al (29) are raising awareness about the necessity of a thorough differential diagnosis process when diagnosing paraganglioma, as it is an extremely rare entity with a high malignant potential. They present the case of a 64 -year-old female with symptoms of bowel obstruction and a palpable abdominal mass with critical markers for paragangliomas, namely Chromogranin A and S100, which should be used as the first step in diagnosis, followed by other valuable immunohistochemical markers. A long-term follow-up is extremely important following the diagnosis of paraganglioma as all these tumors have a malignant potential.

Ilias et al (30) present an original study entitled 'Psychosocial profile of the patients with inflammatory bowel disease', a non-interventional study comprising 60 participants who requested medical advice/check-up as an ambulatory visit or during hospitalization. Their results indicate that psychological factors and different characteristics of IBD patients play a relevant role in the way these patients deal with their disease.

Dindelegan et al (31) demonstrated significant differences between the two types of patients in terms of quality of life in their original study entitled 'Distress in neurocognitive disorders due to Alzheimer's disease and stroke'. 
In their review entitled 'Neuroinflammation and microglia/ macrophage phenotype modulate the molecular background of post-stroke depression: A literature review', Nagy et al (32) highlight the increasing evidence hinting at the central role of neuroinflammation in the development of post-stroke depression.

Șuță et al (33) present 3 cases of presumed sterile endophthalmitis induced by triamcinolone acetonide in three eyes with intermediate noninfectious uveitis. They demonstrate that dense vitreous opacity is a defining factor, the anterior segment inflammation is mild to moderate and a hypopyon is present, which may be a sterile inflammatory reaction or the triamcinolone material itself.

Tăbăcaru et al (34) aimed to investigate the incidence and clinical results of intraoperative flap and interface-related complications after Femtosecond-LASIK surgery, where flap creation was performed with VisuMax ${ }^{\circledR}$ femtosecond laser and conducted a retrospective 10 -year cohort study including all eyes treated for all refractive errors by Femtosecond-LASIK technique. The predictability and safety have improved since the flap incision is assisted by a femtosecond laser, but complications of the flap and interface can still occur during the flap creation. Refractive surgeons should be aware and proper manage any unusual situation.

In 'Accuracy of five intraocular lens formulas in eyes with trifocal lens implant', Mălăescu et al (35) aim to compare the accuracy of intraocular lens (IOL) calculation formulas SRK/T, Hoffer Q, Holladay 1, Haigis and Barrett Universal II in prediction of postoperative refraction for multifocal and implants using a single optical biometry device. Their study demonstrated no statistically significant difference between formulas for this trifocal lens implant. There was a tendency for the refractive prediction error to be within $\pm 0.25 \mathrm{D}$ for most of the eyes with the Haigis formula, and within $\pm 0.50 \mathrm{D}$ for all the eyes with the Barrett Universal II formula in the group with the axial length between 22 and $24.5 \mathrm{~mm}$.

In conclusion, the quality of the studies submitted to the Special Issue of 'Experimental and Therapeutic Medicine' meets the Journal's standards, as it makes fundamental scientific points, thus achieving the proposed aims of the issue. The editor would like to take this opportunity and thank all the authors for their valuable contributions.

\section{References}

1. Tanasa IA, Manciuc C, Carauleanu A, Navolan DB, Bohiltea RE and Nemescu D: Anosmia and ageusia associated with coronavirus infection (COVID-19) - what is known? Exp Ther Med 20: 2344-2347, 2020.

2. Leru PM, Anton VF, Ureche C, Zurac S, Bratu O and Neagoe CD: Mast cell activation syndromes - evaluation of current diagnostic criteria and laboratory tools in clinical practice (Review). Exp Ther Med 20: 2348-2351, 2020.

3. Berghi NO, Dumitru M, Vrinceanu D, Ciuluvica RC, SimioniucPetrescu A, Caragheorgheopol R, Tucureanu C, Cornateanu RS and Giurcaneanu C: Relationship between chemokines and $\mathrm{T}$ lymphocytes in the context of respiratory allergies (Review). Exp Ther Med 20: 2352-2360, 2020.

4. Boldeanu L, Boldeanu MV, Bogdan M, Meca AD, Coman CG, Buca BR, Tartau CG and Tartau LM: Immunological approaches and therapy in burns (Review). Exp Ther Med 20: 2361-2367, 2020.

5. Ciobotaru OR, Stoleriu G, Ciobotaru OC, Grigorovici A, Voinescu DC, Matei MN, Cobzaru RG, Manolache N and Lupu MN: Postanesthetic skin erythema due to succinylcholine versus atracurium. Exp Ther Med 20: 2368-2372, 2020.
6. Sandru F, Popa A, Petca A, Miulescu RG, Constantin MM, Petca RC, Constantin T and Dumitrașcu MC: Etiologic role of Borrelia burgdorferi in morphea: A case report. Exp Ther Med 20: 2373-2376, 2020.

7. Vrinceanu D, Dumitru M, Stefan A, Neagos A, Musat G and Nica EA: Severe DRESS syndrome after carbamazepine intake in a case with multiple addictions: A case report. Exp Ther Med 20: 2377-2380, 2020.

8. Surlin P, Gheorghe DN, Popescu DM, Martu AM, Solomon S, Roman A, Lazar L, Stratul SI, Rusu D, Foia L, Boldeanu MV, Boldeanu L, Danilescu M and Rogoveanu I: Interleukin-1 $\alpha$ and $-1 \beta$ assessment in the gingival crevicular fluid of periodontal patients with chronic hepatitis C. Exp Ther Med 20: 2381-2386, 2020.

9. Bica C, Sandu C, Suceveanu AI, Sarbu E, Stoica RA Gherghiceanu F, Bohiltea RE, Stefan AD and Stoian AP: Non-alcoholic fatty liver disease: A major challenge in type 2 diabetes mellitus (Review): A case report. Exp Ther Med 20: 2387-2391, 2020

10. Papacocea T, Papacocea R, Rădoi M, Pițuru S and Balan DG: Stomach 'tastes' the food and adjusts its emptying: A neurophysiological hypothesis (Review). Exp Ther Med 20: 2392-2395, 2020.

11. Iorga RA, Bacalbasa N, Carsote M, Bratu OG, Stanescu AMA, Bungau S, Pantis C and Diaconu CC: Metabolic and cardiovascular benefits of GLP-1 agonists, besides the hypoglycemic effect (Review). Exp Ther Med 20: 2396-2400, 2020.

12. Laslo CL, Bacalbasa N, Stanescu AMA, Carsote M, Bungau S, Rus M, Bratu OG and Diaconu CC: New oral anticoagulantspossible extension to other indications (Review). Exp Ther Med 20: 2401-2405, 2020.

13. Marcu DR, Iorga L, Diaconu CC, Spinu AD, Mischianu D and Bratu OG: Benefits of erectile function recovery programs after radical prostatectomy (Review). Exp Ther Med 20: 2406-2410, 2020.

14. Voicu DI, Munteanu O, Gherghiceanu F, Arsene LV, Bohiltea RE, Gradinaru DM and Cirstoiu MM: Maternal inherited thrombophilia and pregnancy outcomes. Exp Ther Med 20: 2411-2414, 2020.

15. Turcan N, Bohiltea RE, Ionita-Radu F, Furtunescu F, Navolan D, Berceanu C, Nemescu D and Cirstoiu MM: Unfavorable influence of prematurity on the neonatal prognostic of small for gestational age fetuses. Exp Ther Med 20: 2415-2422, 2020.

16. Bohiltea RE, Zugravu CA, Nemescu D, Turcan N, Paulet FP, Gherghiceanu F, Ducu I and Cirstoiu MM: Impact of obesity on the prognosis of hypertensive disorders in pregnancy. Exp Ther Med 20: 2423-2428, 2020.

17. Păuleț FP, Țurcan N, Gherghiceanu F, Bohîlțea RE, Nemescu D and Cîrstoiu MM: Prognosis of autoimmune thyroid disease associated with hereditary thrombophilia during pregnancy. Exp Ther Med 20: 2429-2433, 2020.

18. NemescuD,TanasaIA,StoianDL,NavolanDB and VinturacheAE: Conservative in utero treatment of fetal dyshormonogenetic goiter with levothyroxine, a systematic literature review. Exp Ther Med 20: 2434-2438, 2020.

19. Gorun F, Motoi S, Malita D, Navolan DB, Nemescu D, Olariu RT, Craina M, Vilibic-Cavlek T, Ciohat I, Boda D and Dobrescu A: Cytomegalovirus seroprevalence in pregnant women in the western region of Romania: A large-scale study. Exp Ther Med 20: 2439-2443, 2020.

20. Uzunov AV, Bohiltea RE, Munteanu O, Nemescu D, Cirstoiu MM: A retrospective study regarding the method of delivery of adolescents in a Romanian Hospital. Exp Ther Med 20: 2444-2448, 2020.

21. Navolan DB, Stoian DL, Bohiltea RE, Crainiceanu Z, Craina ML, Cretu O, Timar B, Vladareanu R, Terness P, Būrger F and Nemescu D: Comparison of early pregnancy serum concentration of neopterin, neopterin/creatinine ratio, C-reactive protein, and chitotriosidase, in pregnant women with birth at term and spontaneous preterm birth. Exp Ther Med 20: 2449-2454, 2020.

22. Olaru OG, Constantin GI and Pena CM: Variation of total serum sialic acid concentration in postmenopausal women. Exp Ther Med 20: 2455-2459, 2020.

23. Iordachescu AC, Cirstoiu MM, Zugravu CA, Teodor OM, Turcan N, Ducu I and Bohiltea RE: Dietary behavior during pregnancy. Exp Ther Med 20: 2460-2464, 2020.

24. Nemescu D, Tanasa IA, Bohiltea RE, Coroleuca C, Bratila E and Vinturache AE: Anatomical and functional changes in arteries of uterine circulation after modified laparoscopic transabdominal cerclage in pregnancy: A case report and review of the literature. Exp Ther Med 20: 2465-2469, 2020. 
25. Zvanca ME, Munteanu A, Bot M, Petca A and Nemescu D Posterior fossa assessment in the axial view of the head at 11-14 weeks of gestation in normal and aneuploid fetuses. Exp Ther Med 20: 2470-2474, 2020.

26. Nemescu D, Adam AM, Tanasa IA, Socolov D, Bohiltea RE Navolan DB and Zvanca ME: Reference ranges for the fetal mesencephalon to occiput measurement at 11 to $13+6$ weeks of gestation. Exp Ther Med 20: 2475-2480, 2020.

27. Movileanu I, Pepa M, Cândea M and Ureche C: Heart valve mechanical prosthesis: The perfect match until it is not - A case report. Exp Ther Med 20: 2481-2483, 2020.

28. Costache RS, Lulache AI, Jinga M, Vlădut C, Costache DO and Radu FI: Use of prokinetics and other quality factors in capsule endoscopy - old and new insights (Review). Exp Ther Med 20: 2484-2488, 2020.

29. Iliesiu A, Ungureanu IA, Petca A, Constantin MM, Petca RC, Sandru F, Constantin T and Dumitrascu MC: Paraganglioma presenting as a mesenteric cystic mass: A case report. Exp Ther Med 20: 2489-2492, 2020.

30. Ilias T, Bungau S, Tit DM, Maghiar D, Hocopan C, Brata R, Bratu OG, Negrut N, Diaconu C and Fratila O: Psychosocial profile of the patients with inflammatory bowel disease. Exp Ther Med 20: 2493-2500, 2020.

31. Dindelegan CM, Faur D, Purza L, Bumbu A and Sabau M: Distress in neurocognitive disorders due to Alzheimer's disease and stroke. Exp Ther Med 20: 2501-2509, 2020.
32. Nagy EE, Frigy A, Szász JA and Horváth E: Neuroinflammation and microglia/macrophage phenotype modulate the molecular background of post-stroke depression: A literature review. Exp Ther Med 20: 2510-2523, 2020.

33. Șuță MC, Karancsi OL, Mușat O, Balica N, Yasar I, Roșca C, Stanca S and Dărăbuş DM: Triamcinolone acetonide induces sterile endophthalmitis in patients with intermediate uveitis: A case report series. Exp Ther Med 20: 2524-2528, 2020.

34. Tăbăcaru B, Stanca S, Mocanu V, Zemba M, Stanca HT and Munteanu M: Intraoperative flap-related complications in FemtoLASIK surgeries performed with Visumax ${ }^{\circledR}$ femtosecond laser: A ten-year Romanian experience. Exp Ther Med 20: 2529-2535, 2020

35. Mălăescu M, Stanca HT, Tăbăcaru B, Stănilă A, Stanca S and Danielescu C: Accuracy of five intraocular lens formulas in eyes with trifocal lens implant. Exp Ther Med 20: 2536-2543, 2020.

This work is licensed under a Creative Commons Attribution-NonCommercial-NoDerivatives 4.0 International (CC BY-NC-ND 4.0) License. 